\title{
Alternative derivations for the Poisson integral formula
}

\author{
J. T. CHEN* and C. S. WU \\ Department of Harbor and River Engineering, \\ National Taiwan Ocean University, Keelung, Taiwan
}

(Received 23 February 2004)

\begin{abstract}
Poisson integral formula is revisited. The kernel in the Poisson integral formula can be derived in a series form through the direct BEM free of the concept of image point by using the null-field integral equation in conjunction with the degenerate kernels. The degenerate kernels for the closed-form Green's function and the series form of Poisson integral formula are also derived. Two and threedimensional cases are considered. Also, interior and exterior problems are both solved. Even though the image concept is required, the location of image point can be determined straightforward through the degenerate kernels instead of the method of reciprocal radii.
\end{abstract}

\section{Introduction}

Integral representation for the solution of partial differential equation is important in both science and engineering. Green's identity and Somigliani identity for the Laplace and Navier problems provide the integral representation, respectively. Based on the integral representation, the boundary integral equation method (BIEM) [11] and the boundary element method (BEM) [1] have been developed to be powerful tools for engineering problems. By choosing the appropriate Green's function, the Poisson integral formulae for the disc and sphere domains subject to the Dirichlet condition were found [9]. How to find the Green's function is not trivial. In the literature, the image point was found in a semi-inverse method in priori through reciprocal radii in the Sommerfeld's book [12] as shown in figure 1. Sommerfeld and Greenberg [9] both utilized the concept of reciprocal radii of William Thomson [13] to derive the Poisson integral formula. To determine the location of image point in a straightforward way is the main concern of the present paper. Also, the possible derivation without employing an image point is also addressed. Fundamental solutions are expanded into degenerate kernels in constructing the Green's function. Since a degenerate kernel separates the source and field points for the closed-form fundamental solution, it plays an important role in studying the rank-deficiency problems analytically in BEM, e.g., degenerate scale [4, 6], spurious eigenvalues $[5,8,10]$ and fictitious frequencies $[2,3]$. Also, it can study the order of pseudo-differential operator [7].

In this paper, the degenerate kernels for the closed-form Green's function and the series form of Poisson integral formula are both derived. The image point is

*Corresponding author. Email: jtchen@mail.ntou.edu.tw 


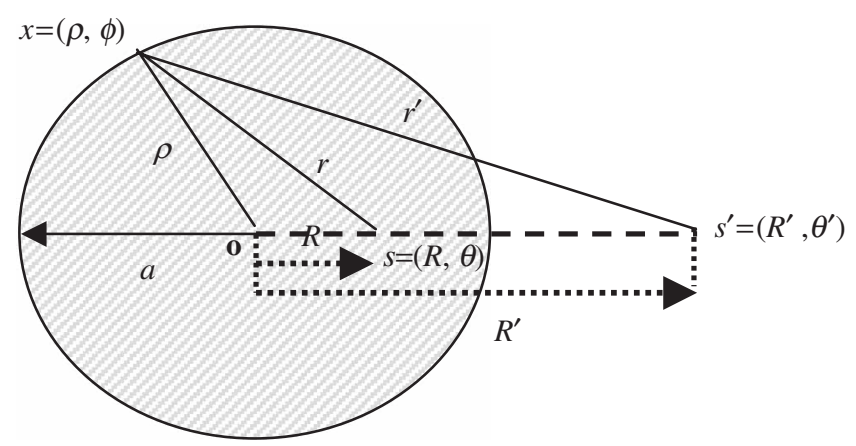

Figure 1. Geometry of reciprocal radii.

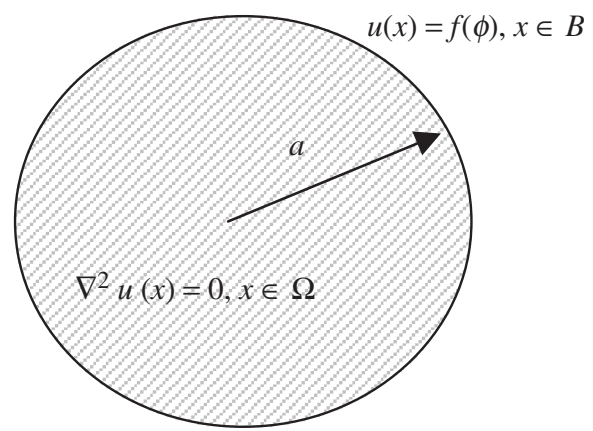

Figure 2. Two-dimensional interior Laplace problem.

determined in a different way to derive the Poisson integral formula by using the degenerate kernels. The closed-form Green's function is expanded into degenerate kernels. Furthermore, the null-field integral equation in conjunction with the degenerate kernel provides us with another approach to derive the Poisson integral formula without using the concept of the image point. Both the two and three dimensional Laplace problems for the exterior problem as well as the interior problem are solved.

\section{Derivation of the Poisson integral formula by using the degenertae scale (two-dimensional problem)}

Let us consider the two-dimensional Laplace equation,

$$
\nabla^{2} u(x)=0, \quad x \in \Omega
$$

where $\nabla^{2}$ is the Laplacian operator, $u(x)$ is the potential and $\Omega$ is the circular domain with radius $a$. For simplicity, we consider the Dirichlet boundary condition, $u=f(\phi)$, as shown in figure 2. By using the fundamental solution as an auxiliary system, Green's identity yields

$$
2 \pi u(s)=\int_{B} T_{F}(x, s) u(x) \mathrm{d} B(x)-\int_{B} U_{F}(x, s) t(x) \mathrm{d} B(x), \quad x \in \Omega
$$


where $u(x)$ and $t(x)$ are the potential and flux, $s$ and $x$ are the source point and field point, respectively, $B$ is the boundary of the domain, $U_{F}$ and $T_{F}$ kernels are

$$
U_{F}(x, s)=\ln (r), \quad T_{F}(x, s)=\frac{\partial U_{F}(x, s)}{\partial n_{x}},
$$

in which $n_{x}$ denotes the normal vector of $x, \nabla^{2} U_{F}(x, s)=\delta(x-s)$ and $r=|x-s|$. By expanding the fundamental solution into the degenerate kernel, the image point can be determined naturally and straightforward to match the homogeneous Dirichlet boundary condition for the Green's function, $U_{G}\left(x ; s, s^{\prime}\right)$ with an image source. Then, we employ the boundary integral equation method (BIEM) to derive the Poisson integral formula. In constructing the Green's function to satisfy the homogeneous Dirichlet boundary condition, image source is required and must be distributed outside the domain. By replacing the $U_{F}$ with $U_{G}$, equation (2) simplifies to

$$
2 \pi u(s)=\int_{B} T_{G}\left(x ; s, s^{\prime}\right) u(x) \mathrm{d} B(x), \quad x \in B,
$$

due to the homogeneous Dirichlet condition of $\left.U_{G}\left(x ; s, s^{\prime}\right)\right|_{x \in B}=0$, where

$$
T_{G}\left(x ; s, s^{\prime}\right)=\frac{\partial U_{G}\left(x ; s, s^{\prime}\right)}{\partial n_{x}} .
$$

Equation (4) indicates that the integral representation solution is expressed in terms of boundary integrals since the kernel of $T_{G}$ and the boundary data of $u(x)$ are both known.

\subsection{2-D interior problem}

Now, we use the degenerate kernels to derive the Green's function and Poisson integral formula in a different way from that of the conventional one $[9,12,13]$. The fundamental solution $U_{F}$ in equation (3) is expanded into

$$
\begin{aligned}
U_{F}(x, s)=\ln (r)=\ln |x-s| & \\
= & \begin{array}{ll}
U_{F}^{I}(x, s)=\ln (R)-\sum_{m=1}^{\infty} \frac{1}{m}\left(\frac{\rho}{R}\right)^{m} \cos [m(\theta-\phi)], & \rho \leq R \\
U_{F}^{E}(x, s)=\ln (\rho)-\sum_{m=1}^{\infty} \frac{1}{m}\left(\frac{R}{\rho}\right)^{m} \cos [m(\theta-\phi)], & \rho \geq R
\end{array}
\end{aligned}
$$

where $x=(\rho, \phi)$ and $s=(R, \theta)$ for the polar coordinate in figure 3 , the superscripts $I$ and $E$ denote the interior and exterior problems, respectively.

Then, we use the method of images and degenerate kernels to derive the closedform and series-form Green's functions. By employing the image method, we locate the image source outside the domain to satisfy the boundary condition as shown in figure 4 . Since $s$ is inside the circle and $x$ is on the boundary to match the boundary condition, we have

$$
\ln |x-s|=\ln (\rho)-\sum_{m=1}^{\infty} \frac{1}{m}\left(\frac{R}{\rho}\right)^{m} \cos [m(\theta-\phi)], \quad \rho>R
$$

Since $s^{\prime}$ is outside the boundary, we have

$$
\ln \left|x-s^{\prime}\right|=\ln \left(R^{\prime}\right)-\sum_{m=1}^{\infty} \frac{1}{m}\left(\frac{\rho}{R^{\prime}}\right)^{m} \cos \left[m\left(\theta^{\prime}-\phi\right)\right], \quad \rho<R^{\prime}
$$




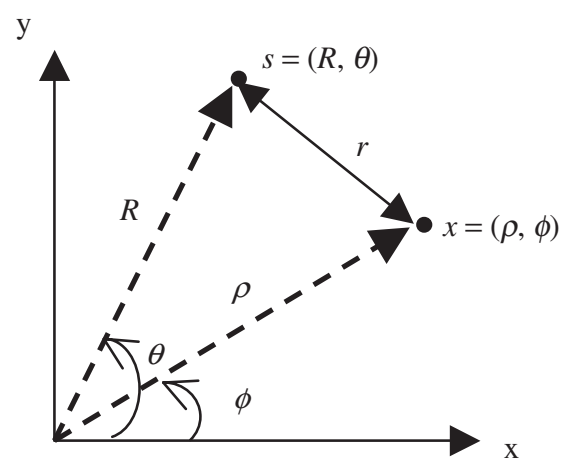

Figure 3. Polar coordinate for the two-dimensional problem.

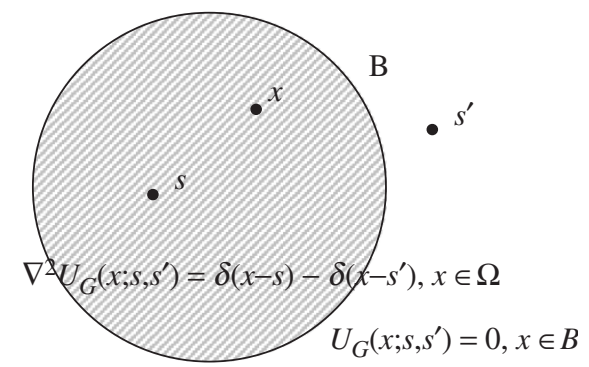

Figure 4. Green's function for the two-dimensional case.

where $s^{\prime}=\left(R^{\prime}, \theta^{\prime}\right)$ is the polar coordinate for the image source $s^{\prime}$ outside the disc as shown in figure 4 . In order to cancel out the $\phi$ term since $\phi$ is arbitrary in the boundary condition, we choose the collinear points $s$ and $s^{\prime}$, i.e., $\theta=\theta^{\prime}$. By equating the $m$ th term in the series of equations (7) and (8), we have

$$
\frac{R}{\rho}=\frac{\rho}{R^{\prime}} \Rightarrow R^{\prime}=\frac{\rho^{2}}{R}=\frac{a^{2}}{R}
$$

By subtracting equation (7) with equation (8), we have

$$
\ln |x-s|-\ln \left|x-s^{\prime}\right|=\ln (a)-\ln \left(R^{\prime}\right)
$$

Since Green's function satisfies the homogeneous Dirichlet boundary conditions, the closed-form Green's function is adjusted to

$$
\begin{aligned}
U_{G}\left(x ; s, s^{\prime}\right) & =\ln |x-s|-\ln \left|x-s^{\prime}\right|-\ln (a)+\ln \left(R^{\prime}\right) \\
& =\ln |x-s|-\ln \left|x-s^{\prime}\right|+\ln (a)-\ln (R)
\end{aligned}
$$

where $\ln (a)$ can be understood as a rigid body term and $\ln (R)$ is the function of $s$ only. The Green's function in equation (11) satisfies

$$
\nabla_{x}^{2} U_{G}\left(x ; s, s^{\prime}\right)=\delta(x-s)-\delta\left(x-s^{\prime}\right), \quad x \in \Omega
$$


(a)

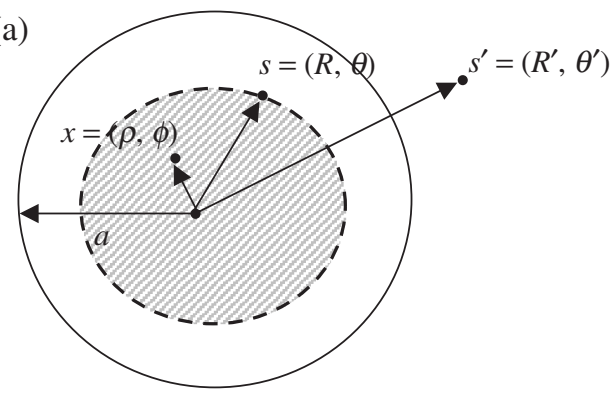

(b)

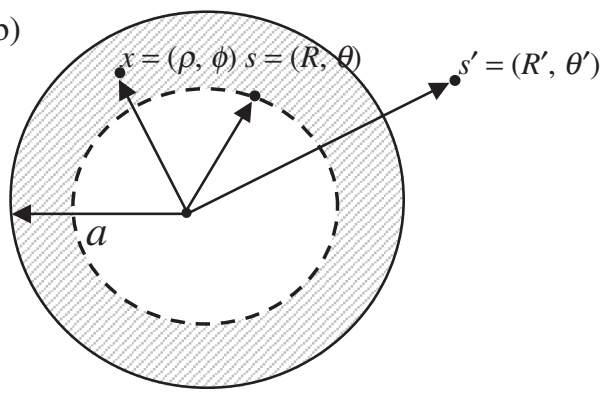

Figure 5. (a) Green's function for the interior problem (core area $0<\rho<R$ ). (b) Green's function for the interior problem (annular area $R<\rho<a$ ).

subject to the boundary condition $\left.U_{G}\left(x ; s, s^{\prime}\right)\right|_{x \in B}=0$. For the circular core $(0<\rho<R)$ in figure 5(a), the closed-form Green's function is expanded into

$$
\begin{aligned}
U_{G}\left(x ; s, s^{\prime}\right)= & \ln |x-s|-\ln \left|x-s^{\prime}\right|+\ln (a)-\ln (R) \\
= & \ln R-\sum_{m=1}^{\infty} \frac{1}{m}\left(\frac{\rho}{R}\right)^{m} \cos [m(\theta-\phi)]-\ln \left(\frac{a^{2}}{R}\right) \\
& +\sum_{m=1}^{\infty} \frac{1}{m}\left(\frac{\rho R}{a^{2}}\right)^{m} \cos [m(\theta-\phi)]+\ln (a)-\ln (R) \\
= & \ln \left(\frac{R}{a}\right)-\sum_{m=1}^{\infty} \frac{1}{m}\left[\left(\frac{\rho}{R}\right)^{m}-\left(\frac{\rho R}{a^{2}}\right)^{m}\right] \cos [m(\theta-\phi)], \quad 0<\rho \leq R
\end{aligned}
$$

The degenerate kernel for the Green's function in the annular region $(R<\rho<a)$ in figure $5(\mathrm{~b})$ is

$$
\begin{aligned}
U_{G}\left(x ; s, s^{\prime}\right)= & \ln |x-s|-\ln \left|x-s^{\prime}\right|+\ln (a)-\ln (R) \\
= & \ln \rho-\sum_{m=1}^{\infty} \frac{1}{m}\left(\frac{R}{\rho}\right)^{m} \cos [m(\theta-\phi)]-\ln \left(\frac{a^{2}}{R}\right) \\
& +\sum_{m=1}^{\infty} \frac{1}{m}\left(\frac{\rho R}{a^{2}}\right)^{m} \cos [m(\theta-\phi)]+\ln (a)-\ln (R) \\
= & \ln \left(\frac{\rho}{a}\right)-\sum_{m=1}^{\infty} \frac{1}{m}\left[\left(\frac{R}{\rho}\right)^{m}-\left(\frac{\rho R}{a^{2}}\right)^{m}\right] \cos [m(\theta-\phi)], \quad R \leq \rho<a
\end{aligned}
$$




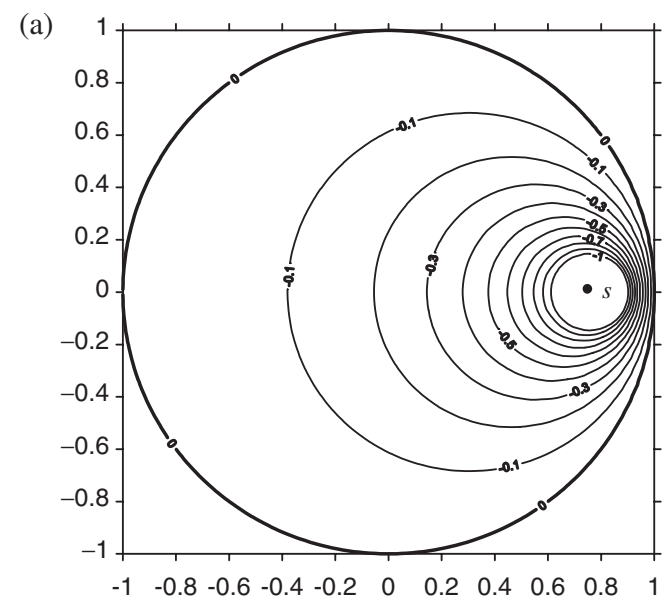

(b)

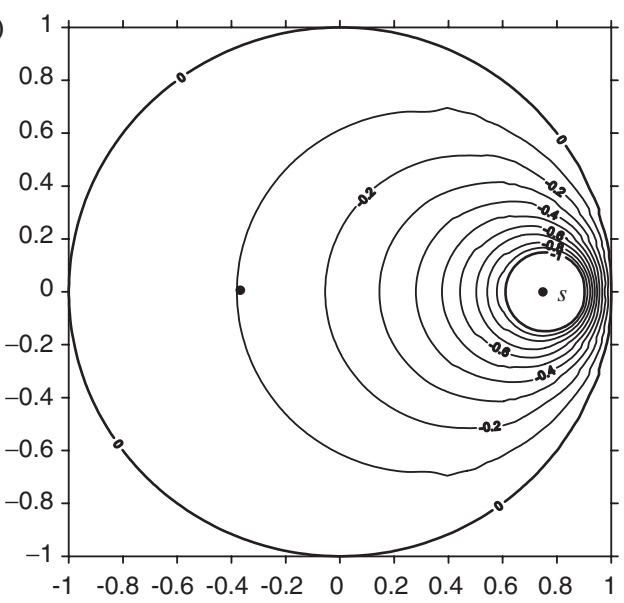

Figure 6. (a) The closed-form Green's function $\left(a=1, R=0.8, R^{\prime}=1.25, \theta=0\right) U_{G}\left(x ; s, s^{\prime}\right)=$ $\ln |x-s|-\ln \left|x-s^{\prime}\right|+\ln a-\ln R$. (b) The series-form Green's function ( $a=1, \quad R=0.8$, $R^{\prime}=1.25, M=50, \theta=0$ )

$$
U_{G}\left(x ; s, s^{\prime}\right)= \begin{cases}\ln \left(\frac{R}{a}\right)-\sum_{m=1}^{M} \frac{1}{m}\left[\left(\frac{\rho}{R}\right)^{m}-\left(\frac{\rho R}{a^{2}}\right)^{m}\right] \cos [m(\theta-\phi)], & 0<\rho \leq R \\ \ln \left(\frac{\rho}{a}\right)-\sum_{m=1}^{M} \frac{1}{m}\left[\left(\frac{R}{\rho}\right)^{m}-\left(\frac{\rho R}{a^{2}}\right)^{m}\right] \cos [m(\theta-\phi)], & R \leq \rho<a .\end{cases}
$$

The closed-form and series-form Green's functions are plotted in figures 6(a) and 6(b), respectively. The series solution using 50 terms in equations (13) and (14) match well with the closed-form solution in equation (11). By substituting equation (11) into equation (4), we obtain the Poisson integral formula

$$
\begin{aligned}
u(R, \theta) & =\frac{1}{2 \pi} \int_{0}^{2 \pi} T_{G}\left(R, \theta ; \rho, \phi ; \frac{a^{2}}{R}, \theta\right) f(\phi) a \mathrm{~d} \phi \\
& =\frac{1}{2 \pi} \int_{0}^{2 \pi} \frac{\left(a^{2}-R^{2}\right)}{\left[a^{2}+R^{2}-2 a R \cos (\phi-\theta)\right]} f(\phi) \mathrm{d} \phi
\end{aligned}
$$




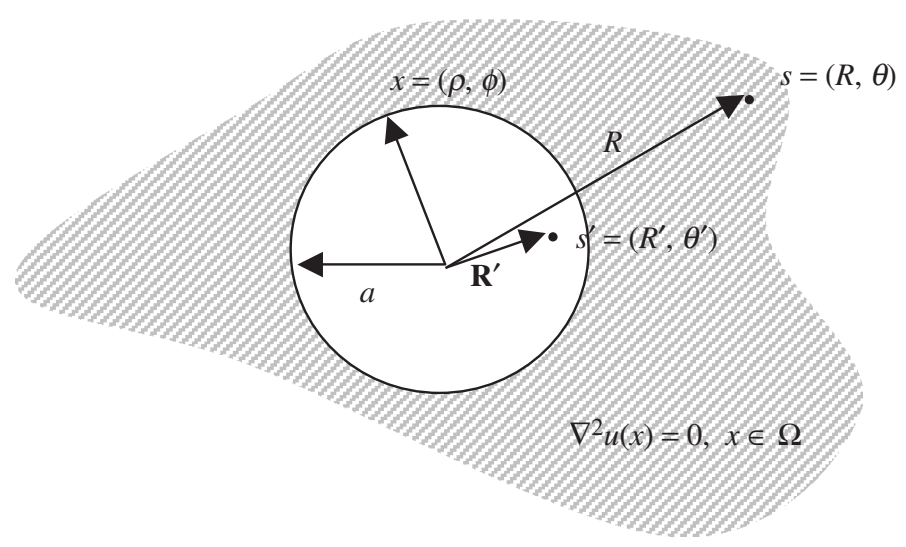

Figure 7. 2-D exterior Laplace problem.

with $0<R<a, 0<\theta<2 \pi$, where $f(\phi)$ is the specified boundary condition. From equation (14), the series-form for the Poisson integral formula is

$$
u(R, \theta)=\frac{1}{2 \pi} \int_{0}^{2 \pi}\left[1+2 \sum_{m=1}^{\infty}\left(\frac{R}{a}\right)^{m} \cos (m(\theta-\phi))\right] f(\phi) \mathrm{d} \phi, \quad 0<R<a, \quad 0<\theta<2 \pi
$$

due to $R<\rho$ in equation (2).

\subsection{2-D exterior problem}

According to the successful experience of the interior problem, we extend it to the exterior problem as shown in figure 7. In a similar way, we have the closed-form Green function for the exterior problem as

$$
U_{G}\left(x ; s, s^{\prime}\right)=\ln |x-s|-\ln \left|x-s^{\prime}\right|+\ln (a)-\ln (R) .
$$

The Green's function satisfies equation (12) and the series form is expressed in two parts. The degenerate kernel for the Green's function (figure 8(a)) in the annular region is

$$
\begin{aligned}
U_{G}\left(x ; s, s^{\prime}\right) & =\ln |x-s|-\ln \left|x-s^{\prime}\right|+\ln (a)-\ln (R) \\
& =\ln \left(\frac{a}{\rho}\right)-\sum_{m=1}^{\infty} \frac{1}{m}\left[\left(\frac{\rho}{R}\right)^{m}-\left(\frac{a^{2}}{\rho R}\right)^{m}\right] \cos [m(\theta-\phi)], \quad a<\rho \leq R .
\end{aligned}
$$

For the unbounded area (figure 8(b)), we have

$$
\begin{aligned}
U_{G}\left(x ; s, s^{\prime}\right) & =\ln |x-s|-\ln \left|x-s^{\prime}\right|+\ln (a)-\ln (R) \\
& =\ln \left(\frac{a}{R}\right)-\sum_{m=1}^{\infty} \frac{1}{m}\left[\left(\frac{R}{\rho}\right)^{m}-\left(\frac{a^{2}}{\rho R}\right)^{m}\right] \cos [m(\theta-\phi)], \quad R \leq \rho<\infty
\end{aligned}
$$


(a)

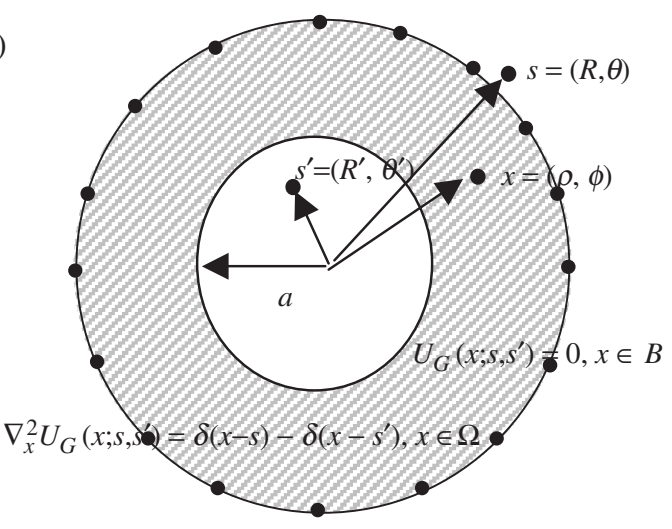

(b)

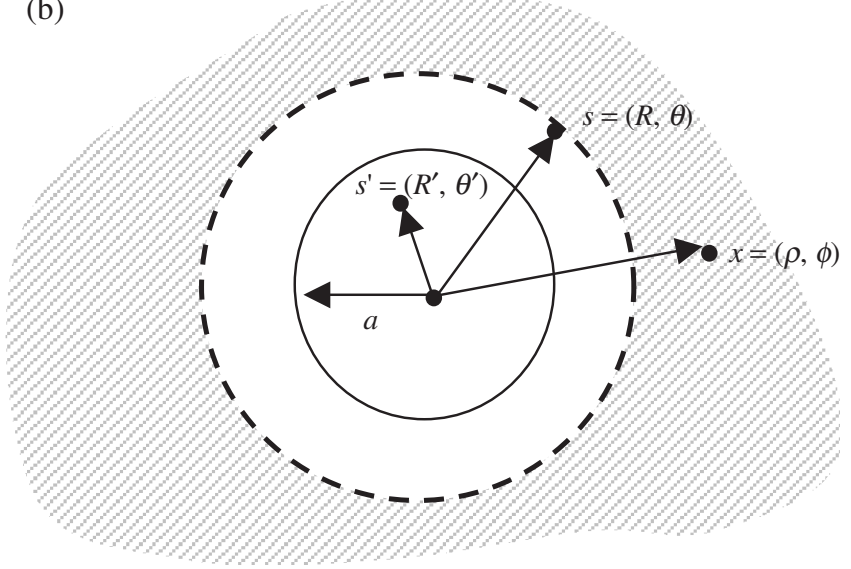

Figure 8. (a) Green's function for the exterior problem (annular region $a<\rho \leq R$ ). (b) Green's function for the exterior problem (unbounded region $R \leq \rho<\infty$ ).

The closed-form and series-form Green's functions are plotted in figures 9(a) and 9(b), respectively. It is found that series solution converges to the exact solution. By using the closed-form Green's function, we have

$$
u(R, \theta)=\frac{1}{2 \pi} \int_{0}^{2 \pi} \frac{\left(R^{2}-a^{2}\right)}{\left[a^{2}+R^{2}-2 a R \cos (\theta-\phi)\right]} f(\phi) \mathrm{d} \phi, \quad a<R<\infty, \quad 0<\theta<2 \pi .
$$

For the series-form Green's function, we have

$$
u(R, \theta)=\frac{-1}{2 \pi} \int_{0}^{2 \pi}\left\{1+2 \sum_{m=1}^{\infty}\left(\frac{a}{R}\right)^{m} \cos (m(\theta-\phi))\right\} f(\phi) \mathrm{d} \phi, \quad a<R<\infty, 0<\theta<2 \pi
$$

due to $\rho<R$ in equation (2). For the two-dimensional interior and exterior problems, we summarize the results in table 1 . 
(a)

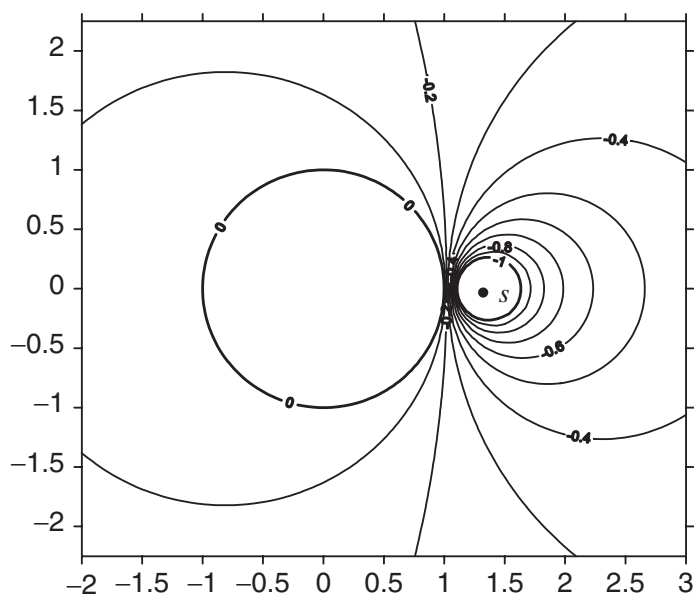

(b)

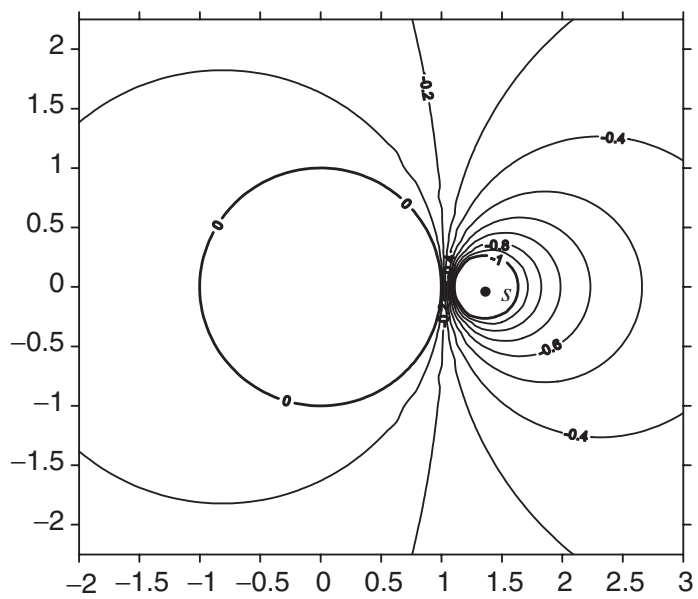

Figure 9. (a) The closed-form Green's function ( $\left.a=1, \quad R=1.25, \quad R^{\prime}=0.8, \quad \theta=0\right)$ $U_{G}\left(x ; s, s^{\prime}\right)=\ln |x-s|-\ln \left|x-s^{\prime}\right|+\ln a-\ln R$. (b) The series-form Green's function $(a=1$, $R=1.25, R^{\prime}=0.8, M=50, \theta=0$ )

$$
U_{G}\left(x ; s, s^{\prime}\right)=\left\{\begin{array}{cl}
\ln \left(\frac{a}{\rho}\right)-\sum_{m=1}^{M} \frac{1}{m}\left[\left(\frac{\rho}{R}\right)^{m}-\left(\frac{a^{2}}{\rho R}\right)^{m}\right] \cos [m(\theta-\phi)], & a<\rho \leq R \\
\ln \left(\frac{a}{R}\right)-\sum_{m=1}^{M} \frac{1}{m}\left[\left(\frac{R}{\rho}\right)^{m}-\left(\frac{a^{2}}{\rho R}\right)^{m}\right] \cos [m(\theta-\phi)], & R \leq \rho<\infty .
\end{array}\right.
$$

\section{Derivation of the Poisson integral formula by using the degenerate kernels (three-dimensional problem)}

By extending 2-D disc to 3-D sphere Laplace problem as shown in figure 10, equation (2) changes to

$$
4 \pi u(s)=\int_{B} T_{F}(x, s) u(x) \mathrm{d} B(x)-\int_{B} U_{F}(x, s) t(x) \mathrm{d} B(x), \quad x \in \Omega
$$


Table 1. Green's function of the two-dimensional Laplace problem.

Problem statement $u(x)=f(\phi)$

Exterior problem

Auxiliary system

Fundamental solution

\section{Green's function}

Poisson integral formula (closed-form)

Poisson integral formula (series-form)
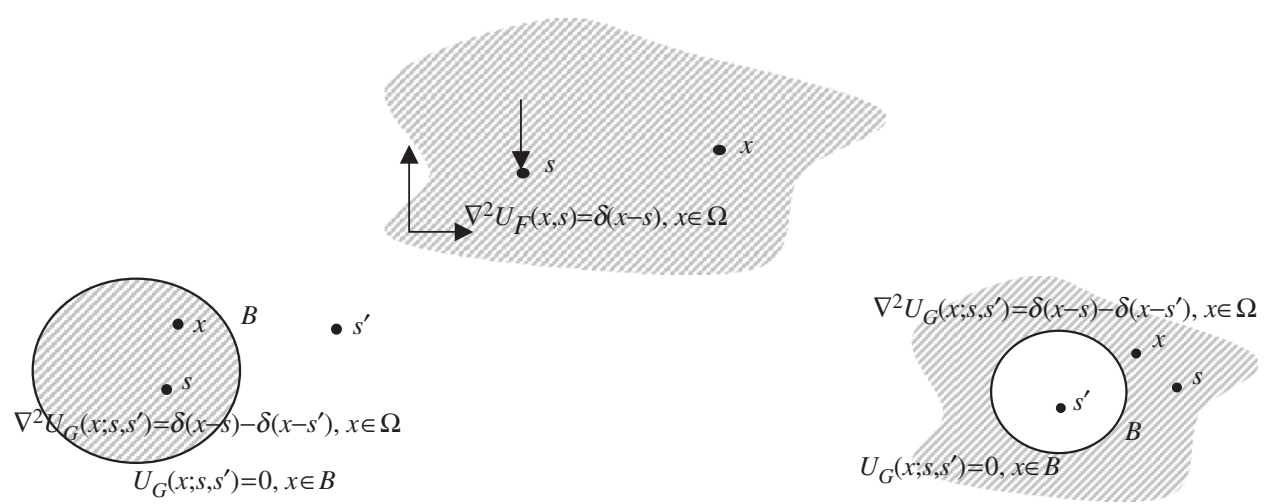

$$
\begin{aligned}
u(R, \theta)= & \frac{1}{2 \pi} \int_{0}^{2 \pi} \frac{\left(R^{2}-a^{2}\right)}{a^{2}+R^{2}-2 a R \cos (\phi-\theta)} f(\phi) \mathrm{d} \phi, \\
& a<R<\infty, 0<\theta<2 \pi
\end{aligned}
$$$$
u(R, \theta)=\frac{-1}{2 \pi} \int_{0}^{2 \pi}\left\{1+2 \sum_{m=1}^{\infty} \frac{a^{m}}{R^{m}} \cos (m(\theta-\phi))\right\} f(\phi) \mathrm{d} \phi,
$$

$u(R, \theta)=\frac{1}{2 \pi} \int_{0}^{2 \pi}\left\{1+2 \sum_{m=1}^{\infty} \frac{R^{m}}{a^{m}} \cos (m(\theta-\phi))\right\} f(\phi) \mathrm{d} \phi$,

$0<R<a, 0<\theta<2 \pi$ 


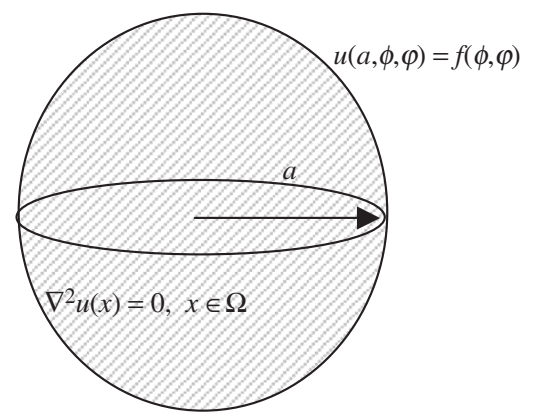

Figure 10. Three-dimensional interior Laplace problem.

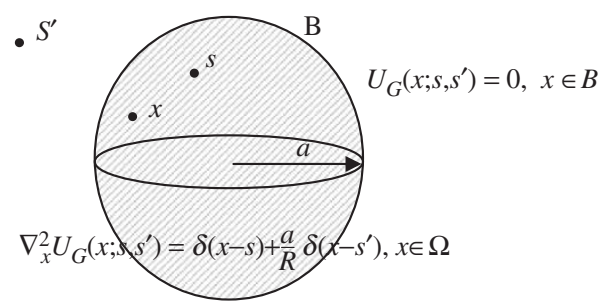

Figure 11. Green's function for the three-dimensional case.

where the fundamental solution $U_{F}$ and the kernel $T_{F}$ are

$$
U_{F}(x, s)=\frac{-1}{r}, \quad T_{F}(x, s)=\frac{\partial U_{F}(x, s)}{\partial n_{x}}
$$

in which $\nabla_{x}^{2} U_{F}(x, s)=\delta(x-s)$ and $r=|x-s|$. In order to derive the Poisson integral formula, an auxiliary system of Green's function subject to the homogeneous Dirichlet boundary condition in figure 11 needs to be constructed first. Equation (4) reduces to

$$
4 \pi u(s)=\int_{B} T_{G}\left(x ; s, s^{\prime}\right) u(x) \mathrm{d} B(x), \quad s \in \Omega,
$$

for the three-dimensional case.

\subsection{3-D interior problem}

We use the degenerate kernel to derive the 3-D closed-form Green's function and the Poisson integral formula. Similarly, the fundamental solution is expressed in a series form as

$$
\begin{aligned}
& U_{F}(x, s)=\frac{-1}{r} \\
& \quad=\left\{\begin{array}{l}
U_{F}^{I}(x, s)=\frac{1}{R}-\sum_{n=1}^{\infty} \sum_{m=0}^{n} \frac{(n-m) !}{(n+m) !} \cos (m(\varphi-\bar{\varphi})) P_{n}^{m}(\cos \phi) P_{n}^{m}(\cos \theta) \frac{\rho^{n}}{R^{n+1}}, \quad R \geq \rho \\
U_{F}^{E}(x, s)=\frac{1}{\rho}-\sum_{n=1}^{\infty} \sum_{m=0}^{n} \frac{(n-m) !}{(n+m) !} \cos (m(\varphi-\bar{\varphi})) P_{n}^{m}(\cos \phi) P_{n}^{m}(\cos \theta) \frac{R^{n}}{\rho^{n+1}}, \quad R \leq \rho .
\end{array}\right.
\end{aligned}
$$




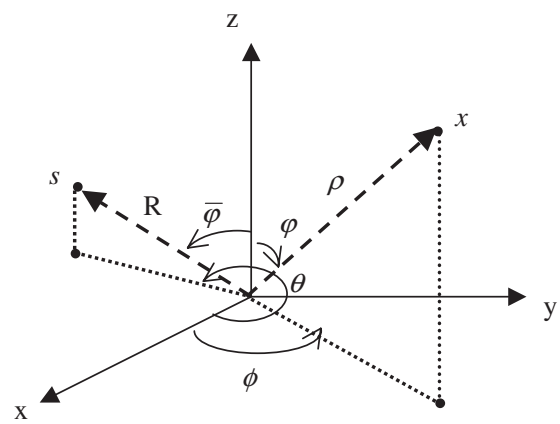

Figure 12. Spherical coordinate for the three-dimensional problem.

where $x=(\rho, \phi, \varphi), s=(R, \theta, \bar{\varphi})$ for the spherical coordinate in figure 12 and $P_{n}^{m}(\cdot)$ is the Legendre function. Then, we use the method of images and degenerate kernel to derive the closed-form and series form Green's functions and locate the image source outside the sphere. Since $s$ is inside the sphere and $x$ is on the boundary to match the boundary condition, we have

$$
\frac{-1}{|x-s|}=\frac{1}{\rho}-\sum_{n=1}^{\infty} \sum_{m=0}^{n} \frac{(n-m) !}{(n+m) !} \cos (m(\varphi-\bar{\varphi})) P_{n}^{m}(\cos \phi) P_{n}^{m}(\cos \theta) \frac{R^{n}}{\rho^{n+1}}, \quad \rho>R
$$

Since $s^{\prime}$ is outside the sphere, we have

$$
\frac{-1}{\left|x-s^{\prime}\right|}=\frac{1}{R^{\prime}}-\sum_{n=1}^{\infty} \sum_{m=0}^{n} \frac{(n-m) !}{(n+m) !} \cos \left(m\left(\varphi-\bar{\varphi}^{\prime}\right)\right) P_{n}^{m}(\cos \phi) P_{n}^{m}\left(\cos \theta^{\prime}\right) \frac{\rho^{n}}{R^{\prime n+1}}, \quad \rho<R^{\prime}
$$

By multiplying equation (27) with $\frac{R^{\prime}}{\rho}$ and subtracting equation (26), the homogeneous Dirichlet boundary condition at $\rho=a$ yields the equal coefficients of the two series,

$$
\frac{R^{n}}{\rho^{n+1}}=\frac{\rho^{n-1}}{\left(R^{\prime}\right)^{n}} \Rightarrow R^{\prime}=\frac{\rho^{2}}{R}=\frac{a^{2}}{R}
$$

on the condition that $s$ and $s^{\prime}$ are collinear $\left(\theta=\theta^{\prime}\right.$ and $\left.\varphi=\bar{\varphi}\right)$. We obtain the closedform Green's function

$$
U_{G}\left(x ; s, s^{\prime}\right)=\frac{-1}{|x-s|}+\frac{a}{R} \frac{1}{\left|x-s^{\prime}\right|}
$$

It is easy to check $\nabla_{x}^{2} U_{G}\left(x ; s, s^{\prime}\right)=\delta(x-s), x \in \Omega$ and $\left.U_{G}\left(x ; s, s^{\prime}\right)\right|_{x \in B}=0$. Similarly, we can express $U_{G}$ into the series form as shown below:

$$
\begin{aligned}
& U_{G}\left(x ; s, s^{\prime}\right) \\
& =\frac{-1}{|x-s|}+\frac{a}{R} \frac{1}{\left|x-s^{\prime}\right|} \\
& = \begin{cases}\frac{1}{R}-\frac{1}{a}+\sum_{n=1}^{\infty} \sum_{m=0}^{n} \frac{(n-m) !}{(n+m) !} \cos (m(\varphi-\bar{\varphi})) P_{n}^{m}(\cos \phi) P_{n}^{m}(\cos \theta)\left[\frac{\rho^{n} R^{n}}{a^{2 n+1}}-\frac{\rho^{n}}{R^{n+1}}\right], & 0<\rho \leq R \\
\frac{1}{\rho}-\frac{1}{a}+\sum_{n=1}^{\infty} \sum_{m=0}^{n} \frac{(n-m) !}{(n+m) !} \cos (m(\varphi-\bar{\varphi})) P_{n}^{m}(\cos \phi) P_{n}^{m}(\cos \theta)\left[\frac{\rho^{n} R^{n}}{a^{2 n+1}}-\frac{R^{n}}{\rho^{n+1}}\right], & R \leq \rho<a\end{cases}
\end{aligned}
$$




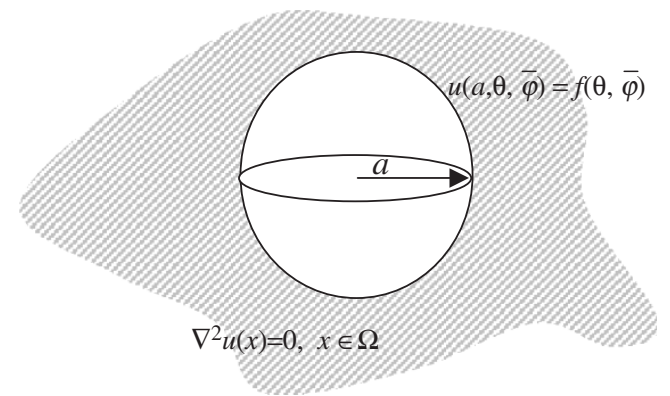

Figure 13. Three-dimensional exterior Laplace problem.

After deriving the closed-form and series-form Green's functions by using the image method, we employ equation (24) to derive the Poisson integral formula as shown below

$$
\begin{aligned}
u(R, \theta, \bar{\varphi}) & =\frac{1}{4 \pi} \int_{B} T_{G}\left(x ; s, s^{\prime}\right) u(x) \mathrm{d} B(x) \\
& =\frac{1}{4} \pi a\left(a^{2}-R^{2}\right) \int_{0}^{\pi} \int_{0}^{2 \pi} \frac{\sin \varphi}{\left(a^{2}+R^{2}-2 a R \cos \gamma\right)^{\frac{3}{2}}} f(\phi, \varphi) \mathrm{d} \phi \mathrm{d} \varphi,
\end{aligned}
$$

with $0<R<a, 0<\theta<2 \pi, 0<\bar{\varphi}<\pi$, where $\cos \gamma=\cos \theta \cos \phi+\sin \theta \sin \phi \times$ $\cos (\varphi-\bar{\varphi}), d B(x)=a^{2} \sin \phi \mathrm{d} \phi \mathrm{d} \varphi$. For the series-form, we have

$$
\begin{aligned}
u(R, \theta, \bar{\varphi})= & \frac{1}{4 \pi} \int_{0}^{\pi} \int_{0}^{2 \pi}\left\{-1+\sum_{n=1}^{\infty} \sum_{m=0}^{n}(2 n+1) \frac{(n-m) !}{(n+m) !} \cos (m(\varphi-\bar{\varphi})) P_{n}^{m}(\cos \phi)\right. \\
& \left.\times P_{n}^{m}(\cos \theta) \frac{R^{n}}{a^{n}}\right\} \sin \varphi f(\phi, \varphi) \mathrm{d} \phi \mathrm{d} \varphi,
\end{aligned}
$$

with $0<R<a, 0<\theta<2 \pi, 0<\bar{\varphi}<\pi$, due to $R<\rho$ in equation (24).

\subsection{3-D exterior problem}

For the 3-D exterior problem in figure 13, we move the image source outside the domain. Similarly, we express the closed-form Green's function into series-form as

$$
\begin{aligned}
U_{G}\left(x ; s, s^{\prime}\right)= & \frac{-1}{|x-s|}+\frac{a}{R} \frac{1}{\left|x-s^{\prime}\right|} \\
= & \left\{\begin{array}{c}
\frac{1}{R}-\frac{a}{R \rho}+\sum_{n=1}^{\infty} \sum_{m=0}^{n} \frac{(n-m) !}{(n+m) !} \cos (m(\varphi-\bar{\varphi})) P_{n}^{m}(\cos \phi) \\
\quad \times P_{n}^{m}(\cos \theta)\left[\frac{a^{2 n+1}}{\rho^{n+1} R^{n+1}}-\frac{\rho^{n}}{R^{n+1}}\right], \quad a<\rho \leq R \\
\frac{1}{\rho}-\frac{a}{R \rho}+\sum_{n=1}^{\infty} \sum_{m=0}^{n} \frac{(n-m) !}{(n+m) !} \cos (m(\varphi-\bar{\varphi})) P_{n}^{m}(\cos \phi) \\
\quad \times P_{n}^{m}(\cos \theta)\left[\frac{a^{2 n+1}}{\rho^{n+1} R^{n+1}}-\frac{R^{n}}{\rho^{n+1}}\right], \quad R \leq \rho<\infty
\end{array}\right.
\end{aligned}
$$


After deriving the closed-form Green's function by using the image method, we can derive the Poisson integral formula by using equation (24) as follows:

$$
\begin{aligned}
u(R, \theta, \bar{\varphi}) & =\frac{1}{4 \pi} \int_{B} T_{G}\left(x ; s, s^{\prime}\right) u(x) \mathrm{d} B(x)=\frac{1}{4 \pi} \int_{B} \frac{\partial U_{G}\left(x ; s, s^{\prime}\right)}{\partial n_{x}} u(x) \mathrm{d} B(x) \\
& =\frac{-1}{4 \pi} a\left(a^{2}-R^{2}\right) \int_{0}^{\pi} \int_{0}^{2 \pi} \frac{\sin \varphi}{\left(a^{2}+R^{2}-2 a R \cos \gamma\right)^{\frac{3}{2}}} f(\phi, \varphi) \mathrm{d} \phi \mathrm{d} \varphi,
\end{aligned}
$$

with $0<R<a, 0<\theta<2 \pi, 0<\bar{\varphi}<\pi$, For the series-form, we have

$$
\begin{aligned}
u(R, \theta, \bar{\varphi})= & \frac{1}{4 \pi} \int_{0}^{\pi} \int_{0}^{2 \pi} \frac{a}{R}\left\{1-\sum_{n=1}^{\infty} \sum_{m=0}^{n}(2 n+1) \frac{(n-m) !}{(n+m) !} \cos (m(\varphi-\bar{\varphi})) P_{n}^{m}(\cos \phi)\right. \\
& \left.\times P_{n}^{m}(\cos \theta) \frac{a^{n}}{R^{n}}\right\} \sin \varphi f(\phi, \varphi) \mathrm{d} \phi \mathrm{d} \varphi,
\end{aligned}
$$

with $a<R<\infty, 0<\theta<2 \pi, 0<\bar{\varphi}<\pi$, due to $R>\rho$ in equation (24). For the three-dimensional interior and exterior problems, we summarize the results in table 2.

\section{Derivation of the Poisson integral formula by using the direct BEM without using the image point}

After deriving the Poisson integral formula by employing the image point in the previous sections, we will derive it by using the direct BEM instead of finding the image point. Then, we employ the null-field integral equation, Fourier series and the degenerate kernels to find the half "unknown" Neumann data. Then, the series-form kernel in the Poisson integral formula can be obtained by using the direct BEM.

\subsection{2-D interior problem}

Let us consider the Laplace problem in equation (1); we employ the direct boundary integral equation method to derive the Poisson integral formula as follows:

$$
2 \pi u(x)=\int_{B} T_{F}^{I}(s, x) u(s) \mathrm{d} B(s)-\int_{B} U_{F}^{I}(s, x) t(s) \mathrm{d} B(s), \quad x \in \Omega
$$

where the superscript $I$ denotes the interior case. By collocating $x$ outside the domain $\left(x \rightarrow B^{+}\right)$as shown in figure 14, we obtain the null-field integral equation

$$
0=\int_{B} T_{F}^{E}(s, x) u(s) \mathrm{d} B(s)-\int_{B} U_{F}^{E}(s, x) t(s) \mathrm{d} B(s), \quad x \in \Omega^{c}
$$

where $\Omega^{c}$ is the complementary domain of $\Omega$. For a circular case, we express the boundary density $u(s)$ and $t(s)$ in terms of the Fourier series

$$
\begin{aligned}
& u(s)=f(\theta)=a_{0}+\sum_{n=1}^{\infty}\left(a_{n} \cos (n \theta)+b_{n} \sin (n \theta)\right), \\
& t(s)=p_{0}+\sum_{n=1}^{\infty}\left(p_{n} \cos (n \theta)+q_{n} \sin (n \theta)\right),
\end{aligned}
$$


Table 2. Green's function of the three-dimensional Laplace problem.

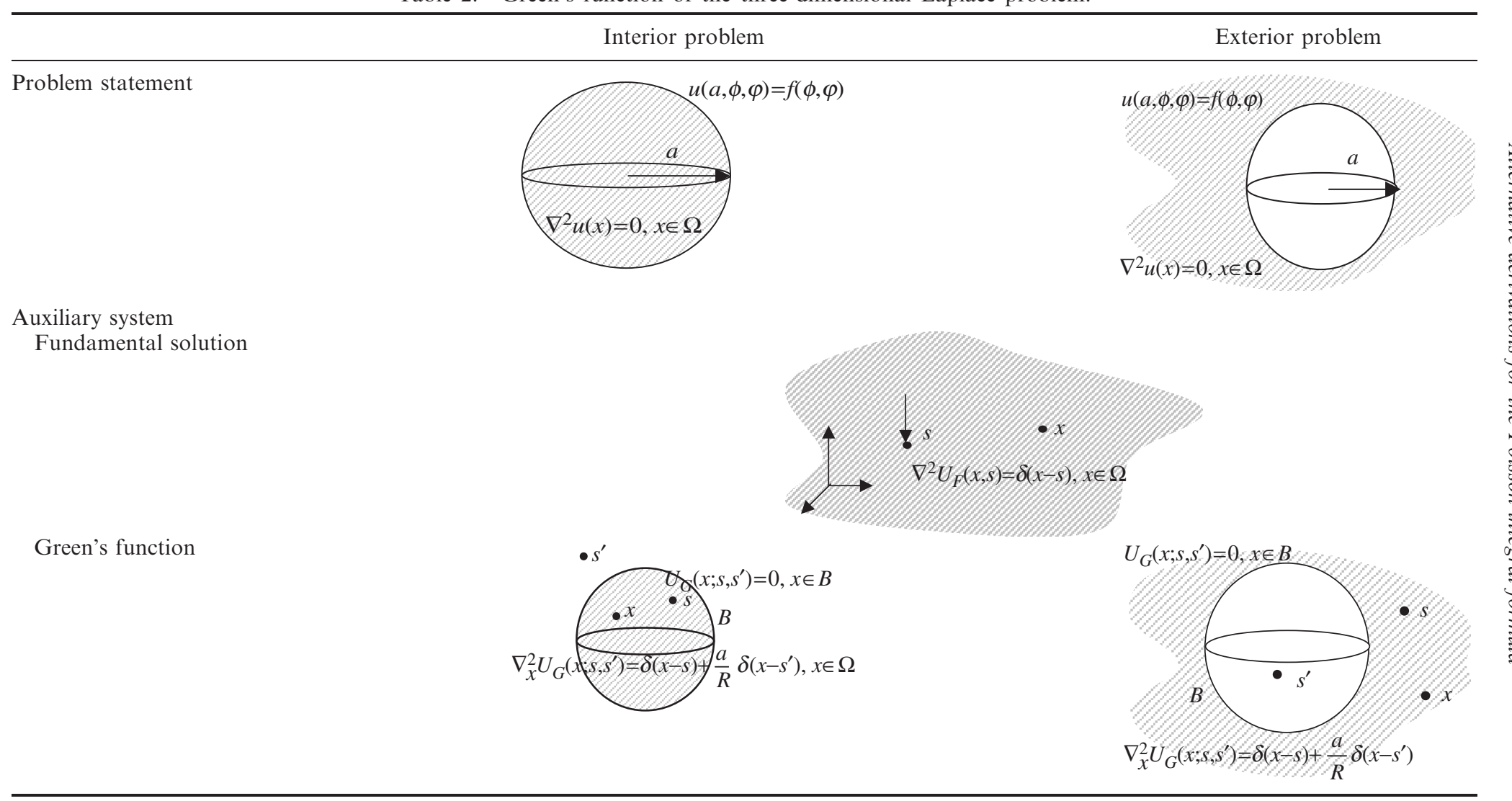


Table 2. Continued.

\begin{tabular}{|c|c|c|}
\hline & Interior problem & Exterior problem \\
\hline $\begin{array}{l}\text { Poisson integral } \\
\text { formula } \\
\text { (closed-form) }\end{array}$ & $\begin{array}{l}u(R, \theta, \bar{\varphi}) \\
=\frac{1}{4 \pi} a\left(a^{2}-R^{2}\right) \int_{0}^{2 \pi} \int_{0}^{\pi} \frac{\sin \varphi}{\left[a^{2}+R^{2}-2 a R \cos \gamma\right]^{\frac{3}{2}}} f(\phi, \varphi) \mathrm{d} \phi \mathrm{d} \varphi \\
0<R<a, 0<\theta<2 \pi, 0<\bar{\varphi}<\pi\end{array}$ & $\begin{aligned} u(R, \theta, \bar{\varphi})= & \frac{-1}{4 \pi} a\left(a^{2}-R^{2}\right) \int_{0}^{2 \pi} \int_{0}^{\pi} \frac{\sin \varphi}{\left[a^{2}+R^{2}-2 a R \cos \gamma\right]^{\frac{3}{2}}} \\
& \times f(\phi, \varphi) \mathrm{d} \phi \mathrm{d} \varphi, \quad a<R<\infty, 0<\theta<2 \pi, 0<\bar{\varphi}<\pi\end{aligned}$ \\
\hline
\end{tabular}




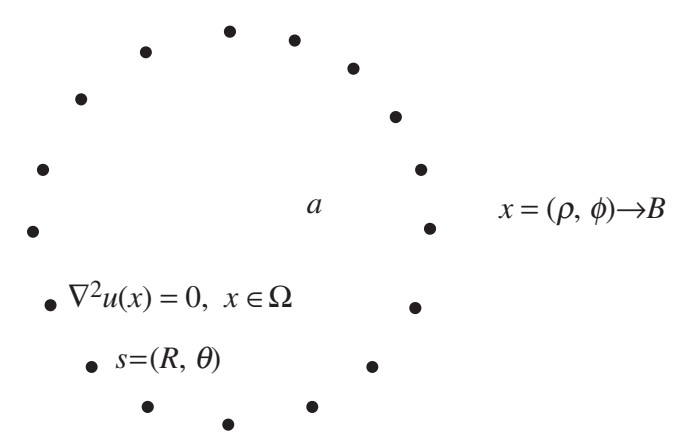

Figure 14. Two-dimensional interior Laplace problem using the null-field integral equation.

where the coefficients $a_{0}, a_{n}$ and $b_{n}$ are specified since $f(\theta)$ is given, $p_{0}, p_{n}$ and $q_{n}$ are to be determined for the Dirichlet problem. Then, we expand the $U_{F}$ kernel in equation (6) and the $T_{F}$ kernel is

$$
T_{F}(s, x)=\frac{\partial U_{F}(s, x)}{\partial n_{s}}= \begin{cases}T_{F}^{I}(s, x)=\frac{1}{R}+\sum_{m=1}^{\infty} \frac{\rho^{m}}{R^{m+1}} \cos (m(\theta-\phi)), & \rho<R \\ T_{F}^{E}(s, x)=-\sum_{m=1}^{\infty} \frac{R^{m-1}}{\rho^{m}} \cos (m(\theta-\phi)), & \rho>R .\end{cases}
$$

By substituting the degenerate kernels of $U_{F}^{E}(s, x)$ and $T_{F}^{E}(s, x)$ when $\rho>R$ and equations (38) and (39) into equation (37) for $x \in B^{+}$, we obtain the unknown coefficients as follows:

$$
\begin{aligned}
p_{0} & =0, \\
p_{m} & =\frac{m}{a} a_{m}, \\
q_{m} & =\frac{m}{a} b_{m}
\end{aligned}
$$

The boundary density of $t(s)$ can be obtained as

$$
t(s)=\sum_{m=1}^{\infty} \frac{m}{a}\left(a_{m} \cos (m \theta)+b_{m} \sin (m \theta)\right)
$$

By substituting equation (42) and the degenerate kernels as well as boundary condition of equation (39) into the equation (36), we have

$$
\begin{aligned}
2 \pi u(x)= & \int_{0}^{2 \pi}\left[\frac{1}{a}+\sum_{m=1}^{\infty} \frac{\rho^{m}}{a^{m+1}} \cos (m(\theta-\phi))\right]\left[a_{0}+\sum_{m=1}^{\infty}\left(a_{m} \cos (m \theta)+b_{m} \sin (m \theta)\right)\right] a \mathrm{~d} \theta \\
& -\int_{0}^{2 \pi}\left[\ln a-\sum_{m=1}^{\infty} \frac{1}{m}\left(\frac{\rho}{a}\right)^{m} \cos (m(\theta-\phi))\right]\left[\sum_{m=1}^{\infty} \frac{m}{a}\left(a_{m} \cos (m \theta)+b_{m} \sin (m \theta)\right)\right] a \mathrm{~d} \theta
\end{aligned}
$$




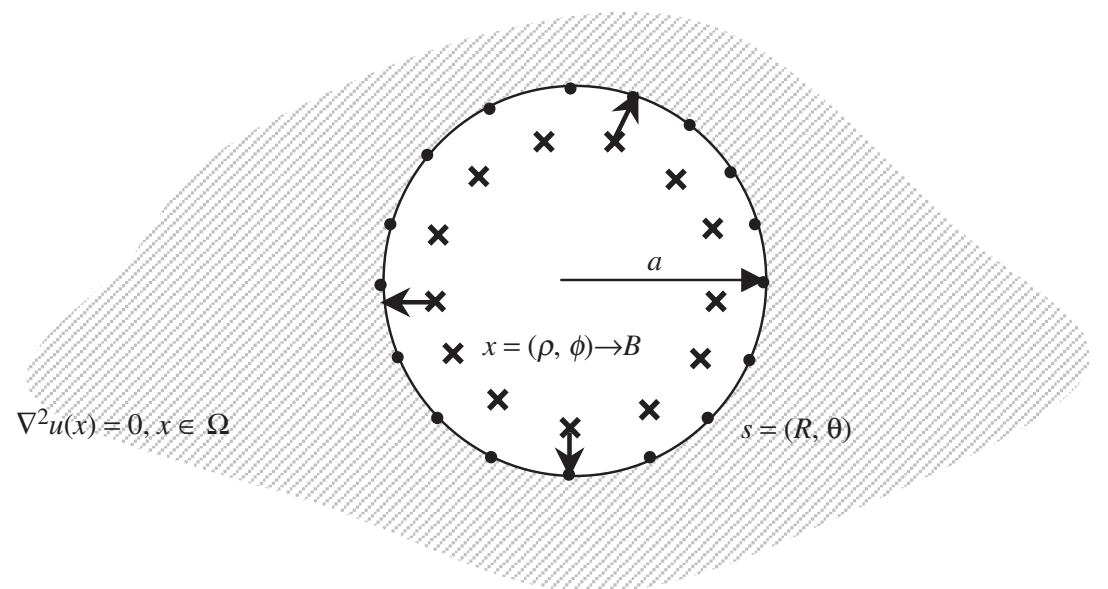

Figure 15. Two-dimensional exterior Laplace problem using the null-field integral equation.

Equation (43) yields the series-form Poisson integral formula

$$
u(\rho, \phi)=\frac{1}{2 \pi} \int_{0}^{2 \pi}\left[1+2 \sum_{m=1}^{\infty}\left(\frac{\rho}{a}\right)^{m} \cos (m(\theta-\phi))\right] f(\theta) \mathrm{d} \theta
$$

Equation (44) is found to be the same of equation (16) without employing any idea of image point.

\subsection{2-D exterior problem}

Based on the successful experience in the interior problem, we extend it into the exterior case as shown in figure 15 and have the boundary integral equation

$$
2 \pi u(x)=\int_{B} T_{F}^{E}(s, x) u(s) \mathrm{d} B(s)-\int_{B} U_{F}^{E}(s, x) t(s) \mathrm{d} B(s), \quad x \in \Omega
$$

By collocating $x$ outside the domain $\left(x \rightarrow B^{-}\right)$, we obtain the null-field integral equation

$$
0=\int_{B} T_{F}^{I}(s, x) u(s) \mathrm{d} B(s)-\int_{B} U_{F}^{I}(s, x) t(s) \mathrm{d} B(s), \quad x \in \Omega^{c}
$$

Similarly, we substitute the degenerate kernels $(\rho<R)$ and the boundary densities in equations (38) and (39) into equation (46) and obtain the coefficients

$$
\begin{aligned}
p_{0} & =\frac{1}{a \ln (a)} a_{0}, \\
p_{m} & =-\frac{m}{a} a_{m}, \\
q_{m} & =-\frac{m}{a} b_{m}
\end{aligned}
$$


The boundary density $t(s)$ is expressed in terms of

$$
t(s)=\frac{a_{0}}{a \ln (a)}-\sum_{m=1}^{\infty} \frac{m}{a}\left(a_{m} \cos (m \theta)+b_{m} \sin (m \theta)\right)
$$

By substituting the degenerate kernels and boundary densities of equations (38) and (48) into equation (45), we have the series-form Poisson integral formula as follows:

$$
u(\rho, \phi)=\frac{-1}{2 \pi} \int_{0}^{2 \pi}\left[\frac{\ln (\rho)}{\ln (a)}+2 \sum_{m=1}^{\infty}\left(\frac{a}{\rho}\right)^{m} \cos (m(\theta-\phi))\right] f(\theta) \mathrm{d} \theta
$$

After comparing equation (49) with equation (21), we may wonder that the kernels are different. However, this does not break the equivalence between equations (21) and (49) since

$$
\int_{B} f(\theta) \mathrm{d} \theta=0
$$

due to the vanishing potential at infinity.

\subsection{3-D interior problem}

By extending 2-D disc to 3-D sphere Laplace problem, we have the boundary integral equation

$$
4 \pi u(x)=\int_{B} T_{F}^{I}(s, x) u(s) \mathrm{d} B(s)-\int_{B} U_{F}^{I}(s, x) t(s) \mathrm{d} B(s), \quad x \in \Omega
$$

Similarly, the boundary densities $u(s)$ and $t(s)$ are expanded into spherical harmonics

$$
\begin{aligned}
& u(s)=f(\theta, \bar{\varphi})=\sum_{n=0}^{\infty} \sum_{m=0}^{n} \alpha_{m n} P_{n}^{m}(\cos \theta) \cos (n \bar{\varphi}), \\
& t(s)=\sum_{n=0}^{\infty} \sum_{m=0}^{n} \beta_{m n} P_{n}^{m}(\cos \theta) \cos (n \bar{\varphi})
\end{aligned}
$$

where $\alpha_{m n}$ is specified and $\beta_{m n}$ is to be determined. Then, we expand the $U_{F}$ kernel in equation (25) and the $T_{F}$ kernel is

$$
\begin{aligned}
& T_{F}(s, x)=\frac{\partial U_{F}(s, x)}{\partial n_{s}} \\
& =\left\{\begin{array}{l}
T_{F}^{I}(s, x)=\frac{-1}{R^{2}}+\sum_{n=1}^{\infty} \sum_{m=0}^{n} \frac{(n-m) !}{(n+m) !}(n+1) \cos (m(\varphi-\bar{\varphi})) P_{n}^{m}(\cos \phi) P_{n}^{m}(\cos \theta) \frac{\rho^{n}}{R^{n+2}} \\
\rho<R \\
T_{F}^{E}(s, x)=\sum_{n=1}^{\infty} \sum_{m=0}^{n} \frac{(n-m) !}{(n+m) !}(-n) \cos (m(\varphi-\bar{\varphi})) P_{n}^{m}(\cos \phi) P_{n}^{m}(\cos \theta) \frac{R^{n-1}}{\rho^{n+1}} \\
\rho>R
\end{array}\right.
\end{aligned}
$$


By substituting the degenerate kernels $(R>\rho)$ and the boundary densities of equations (52) and (53) into the null-field integral equation for $x \in B^{+}$, we obtain the undetermined coefficients

$$
\beta_{m n}=\frac{n}{a} \alpha_{m n}
$$

where $n=0,1, \ldots, \infty$ and $m=0,1, \ldots, n$. The boundary density $t(s)$ is rewritten as

$$
t(s)=\sum_{n=0}^{\infty} \sum_{m=0}^{n} \frac{n}{a} \alpha_{m n} P_{n}^{m}(\cos \theta) \cos (n \bar{\varphi})
$$

Substituting the degenerate kernels $(R>\rho)$ and the boundary densities of equations (52) and (56) into equation (51), we have the series-form Poisson integral formula,

$$
\begin{aligned}
u(\rho, \phi)= & \frac{1}{4 \pi} \int_{0}^{\pi} \int_{0}^{2 \pi}\left[-1+\sum_{n=1}^{\infty} \sum_{m=0}^{n} \frac{(n-m) !}{(n+m) !} \cos (m(\varphi-\bar{\varphi}))\right. \\
& \left.\times P_{n}^{m}(\cos \phi) P_{n}^{m}(\cos \theta)(2 n+1) \frac{\rho^{n}}{a^{n}}\right] \sin \bar{\varphi} f(\theta, \bar{\varphi}) \mathrm{d} \theta \mathrm{d} \bar{\varphi} .
\end{aligned}
$$

Equation (57) is found to be the same of equation (32) without employing any idea of image point.

\subsection{3-D exterior problem}

For the three-dimensional problem, we have the boundary integral equation

$$
4 \pi u(x)=\int_{B} T_{F}^{E}(s, x) u(s) \mathrm{d} B(s)-\int_{B} U_{F}^{E}(s, x) t(s) \mathrm{d} B(s), \quad x \in \Omega
$$

We use the null-field integral equation and degenerate kernels to derive the Poisson integral formula. By the same way, we obtain the the undetermined coefficients as follows:

$$
\beta_{m n}=\frac{-(n+1)}{a} \alpha_{m n}
$$

where $n=0,1, \ldots, \infty$ and $m=0,1, \ldots, n$. The boundary density of $t(s)$ is

$$
t(s)=\sum_{n=0}^{\infty} \sum_{m=0}^{n} \frac{-(n+1)}{a} \alpha_{m n} P_{n}^{m}(\cos \theta) \cos (n \bar{\varphi})
$$

Substituting the degenerate kernels $(R<\rho)$ and the boundary densities of equations (52) and (60) into equation (58), we have the series-form Poisson integral formula,

$$
\begin{aligned}
& u(\rho, \phi) \\
& =\frac{-1}{4 \pi} \int_{0}^{\pi} \int_{0}^{2 \pi}\left[-1+\sum_{n=1}^{\infty} \sum_{m=0}^{n} \frac{(n-m) !}{(n+m) !} \cos (m(\varphi-\bar{\varphi})) P_{n}^{m}(\cos \phi) P_{n}^{m}(\cos \theta)(2 n+1) \frac{a^{n+1}}{\rho^{n+1}}\right] \\
& \quad \times \sin \bar{\varphi} f(\theta, \bar{\varphi}) \mathrm{d} \theta \mathrm{d} \bar{\varphi} .
\end{aligned}
$$

After comparing equation (61) with equation (35), we may doubt the difference of kernels. The reason is the same as equation (50). 


\section{Conclusions}

Alternative ways to derive the Poisson integral formula were proposed in this paper. The closed-form Green's function was expanded into degenerate kernels. Not only interior problems but also exterior problems were examined to check the validity of the present formulation for both 2-D and 3-D Laplace problems. By employing the null-field integral equation in conjunction with the degenerate kernel and Fourier expansion, the series-form kernel in the Poisson integral formula was derived through the direct BEM free of the concept of the image point. Even though the conventional method of image is used, we determined the location of image in a different way.

\section{References}

[1] Banerjee, P.K. and Butterfield, R., 1994, Boundary Element Method in Engineering Science (London: McGraw-Hill).

[2] Chen, J.T., 1998, On the fictitious frequencies using dual series representation. Mech. Res. Comm., 25, 529-534.

[3] Chen, J.T. and Kuo, S.R., 2000, On fictitious frequency using circulants for radiation problem of a cylinder. Mech. Res. Comm., 27(1), 49-58.

[4] Chen, J.T., Lin, J.H., Kuo, S.R. and Chiu, Y.P., 2001, Analytical study and numerical experiments for degenerate scale problems in boundary element method using degenerate kernels and circulants. Engng. Ana. Bound. Elem., 25(9), 819-828.

[5] Chen, J.T., Lin, J.H., Kuo, S.R. and Chyuan, S.W. 2001, Boundary element analysis for the Helmholtz eigenvalue problems with a multiply-connected domain. Proc. R. Soc. Lond. A, 457, 2521-2546.

[6] Chen, J.T., Lee, C.F., Chen, I.L. and Lin, J.H., 2002, An alternative method for degenerate scale problem in boundary element methods for the two-dimensional Laplace equation. Engng. Ana. Bound. Elem., 26(7), 559-569.

[7] Chen, J.T. and Chiu, Y.P., 2002, On the pseudo-differential operators in the dual boundary integral equations using degenerate kernels and circulants. Engng. Ana. Bound. Elem., 26, 41-53.

[8] Chen, J.T., Liu, L.W. and Hong, H.-K., 2003, Spurious and true eigenvalues of Helmholtz BIEs and BEMs for a multiply-connected problem. Proc. Royal Soc. London Ser. A, 459, 1891-1924.

[9] Greenberg, M.D., 1971, Application of Green's Functions in Science and Engineering (New Jersey: Prentice-Hill).

[10] Kuo, S.R., Chen, J.T., Huang, C.X., 2000, Analytical study and numerical experiments for true and spurious eigensolutions of a circular cavity using the real-part BEM. Int. J. Numer. Meth. Engng., 48, 1401-1422.

[11] Liggett, J.A. and Liu, P.L.-F., 1983, The Boundary Integral Equation Methods for Porous Media Flow (London: George Allen and Unwin).

[12] Sommerfeld, A., 1949, Partial Differential Equations in Physics (New York: Academic Press).

[13] Thomson, W., 1848, Maxwell in His Treatise, Vol. I., Chap. XI, quotes a paper in the Cambridge and Dublin Math. Journ. of 1848 (New York: Oxford University Press). 